\title{
COMMENTARY
}

\section{Endothelin antagonists in subarachnoid hemorrhage: what next?}

\author{
R Loch Macdonald ${ }^{* 1,2,3}$ \\ See related research by Ma et al., http://ccforum.com/content/16/5/R198
}

\begin{abstract}
In the previous issue of Critical Care, Ma and colleagues perform a meta-analysis of five randomized, clinical trials of endothelin antagonists in patients with aneurysmal subarachnoid hemorrhage. There are four trials using clazosentan and one trial with TAK-044. These studies show that endothelin plays an important role in the genesis of angiographic vasospasm. The benefit of these drugs is less on delayed cerebral ischemia and nonexistent on overall clinical outcome. Why the drugs reduce vasospasm but do not improve outcome could be because of side effects such as hypotension and pulmonary complications that are more common in patients treated with endothelin antagonists or because rescue therapy, which is used more in the placebo groups, improves outcome in these patients to the same extent as the endothelin antagonists. As the authors conclude, future studies of these drugs will need to consider these and other factors in their design.
\end{abstract}

In the previous issue of Critical Care, Ma and colleagues report a meta-analysis of the randomized, clinical trials of clazosentan and TAK-044 in patients with aneurysmal subarachnoid hemorrhage (SAH) [1]. The endothelins (ETs) are a family of three (ET-1, ET-2 and ET-3) 21-amino-acid peptides that act on several receptors, principally $\mathrm{ET}_{\mathrm{A}}$ and $\mathrm{ET}_{\mathrm{B}}$ receptors in the vasculature. The main effect is to cause vasoconstriction. Experimental data as well as the results of this meta-analysis show that this system is important in the pathogenesis of angiographic vasospasm after SAH [2]. A variety of ET receptor antagonists have been developed. Clazosentan is

*Correspondence: macdonaldlo@smh.ca

'Division of Neurosurgery, St. Michael's Hospital, University of Toronto,

30 Bond Street, Toronto, Ontario, Canada, M5B 1W8

Full list of author information is available at the end of the article a heteroarylsulfonamido pyrimidine that was specifically developed to be a relatively water-soluble, small-molecule, highly-selective $\mathrm{ET}_{\mathrm{A}}$ receptor antagonist for prevention of angiographic vasospasm [3]. TAK-044 is a relatively nonselective antagonist of $\mathrm{ET}_{\mathrm{A}}$ and $\mathrm{ET}_{\mathrm{B}}$ receptors [4].

$\mathrm{Ma}$ and colleagues identified five randomized clinical trials of ET antagonists for SAH. Their meta-analysis gives the same results as the trials, which at least for the four largest studies all had basically the same results. The pooled relative risk (RR) of angiographic vasospasm with ET antagonist treatment was 0.66 (95\% confidence interval $(\mathrm{CI})=0.57$ to 0.77 ), so these drugs effectively reduce vasospasm. The main consequence of vasospasm, delayed cerebral ischemia (DCI), was defined in the last three clazosentan studies as delayed ischemic neurological deficit. The current meta-analysis reports delayed ischemic neurological deficit and DCI, but DCI is defined as infarction on computed tomography 'only attributable to cerebral vasospasm and DCI' [1], which is a partly circular definition. These varied definitions lead to confusion since the definitions vary in the studies and the terminology of $\mathrm{Ma}$ and colleagues does not match that recommended by Vergouwen and colleagues [5]. Interestingly, and not unexpectedly, there was a significant reduction in delayed ischemic neurological deficit $(\mathrm{RR}=0.77,95 \% \mathrm{CI}=0.66$ to 0.90$)$ and a trend towards reduction in DCI $(\mathrm{RR}=0.87,95 \% \mathrm{CI}=0.74$ to 1.03$)$. Despite these improvements, there was no effect on mortality and unfavorable outcome. Thus, considering the pathway from angiographic vasospasm to ischemia (DCI by most definitions), to infarction, and to poor outcome, the benefits of ET antagonists diminish at each step.

The findings of $\mathrm{Ma}$ and colleagues are virtually identical to a meta-analysis conducted by Vergouwen and colleagues [6]. Vergouwen and colleagues, however, also reported data from a subset of the studies showing that there was no significant reduction in vasospasm-related cerebral infarction ( $R R=0.76,95 \% \mathrm{CI}=0.53$ to 1.11 ) although the $R R$ is reduced, in keeping with the analysis of $\mathrm{Ma}$ and colleagues. ET antagonists did not seem to have any effect on all new cerebral infarction $(R R=1.04 ; 95 \%$ 
$\mathrm{CI}=0.91$ to 1.19$)$. This is an important finding since cerebral infarction is one of the most important prognostic factors for outcome after SAH. The odds ratios show the same pattern as mentioned above.

Why is there a substantial effect on angiographic vasospasm, less effect on infarction judged to be due to vasospasm and no effect on all delayed infarcts and clinical outcome? One theory is that the delayed infarctions are not due solely to angiographic vasospasm. This theory predicts that reducing angiographic vasospasm may not be adequate to reduce infarction and improve outcome. Under this theory, the vasospasm-related and any new infarction incidences should be the same. Strictly speaking, they are the same - although, as noted above, the trends in the odds ratios seem different. One alternative theory is that side effects of the drugs, such as hypotension and pulmonary complications, counteract the beneficial effects of reducing vasospasm so that there is no overall beneficial effect on outcome. Indeed, both meta-analyses report virtually identical and significant increases in lung complications, hypotension and anemia in the patients treated with ET antagonists. To fit the data, this theory would require those side effects being sufficient to cause infarctions so that the overall infarction rate is about the same. One could argue that the data, while not conclusive, favor the second theory. Another fundamental issue is that patients in the placebo groups of these studies are administered rescue therapies for DCI in a higher percentage of cases than in the drugtreated groups. If rescue therapy is efficacious, then this also could reduce the difference between the groups in cerebral infarction and overall clinical outcome.

What are some of the limitations of Ma and colleagues' meta-analysis? The strengths of the current analysis are that it is rigorous and follows preferred reporting items for systematic review and meta-analysis (Preferred Reporting Items for Systematic reviews and MetaAnalyses) guidelines [7]. The results of this meta-analysis are not surprising, given that the results for all of the individual studies are the same - which is not a weakness but a comment. Another issue is the inclusion of drugs of different chemical classes and with different known pharmacologic actions in these sorts of meta-analyses. Multiple doses and methods and timing of administration of different drugs are combined into single treatment groups, which makes no sense biologically.

What does the future hold for ET antagonists in SAH? Since all of the studies are only recently completed, obtaining the individual patient data from the sponsors may allow further analysis to guide further studies. This collation is obviously being done, since Actelion (Allschwill, Switzerland) sponsored all of the clazosentan studies, have the data and have invested heavily in clazosentan. According to Vergouwen and colleagues,
Actelion did not provide individual patient data or data that would enable an intention-to-treat analysis [6]. The former omission is an issue. The latter missing data, however, given the small number of patients involved, are not going to change the overall findings. Actelion, however, must be complimented for supporting development of clazosentan and for conducting these studies that would not have occurred if we waited for funding from peer-reviewed granting agencies. The studies they have conducted have been fundamentally directed at improving the outcome of patients with SAH and there cannot be any question about their motivation to develop a drug that will address this.

In summary, the authors' conclusion is that future studies of ET antagonists should be 'more carefully formulated and designed'. Input into the design of these studies would be welcome, given that all of these studies were already very carefully formulated and designed. My opinion is that some method of reducing the side effects of ET antagonists, primarily hypotension and pulmonary complications, is the key to the future of these drugs.

\section{Abbreviations}

$\mathrm{Cl}$, confidence interval; DCl, delayed cerebral ischemia; ET, endothelin; RR, relative risk; $\mathrm{SAH}$, subarachnoid hemorrhage.

\section{Competing interests}

RLM is a consultant for Actelion Pharmaceuticals, Chief Scientific Officer of Edge Therapeutics Inc., and holds patents related to the prevention of delayed cerebral ischemia after subarachnoid hemorrhage.

\section{Acknowledgements}

RLM receives grant support from the Physicians Services Incorporated Foundation, the Brain Aneurysm Foundation, the Canadian Institutes of Health Research and the Heart and Stroke Foundation of Canada.

\section{Author details}

'Division of Neurosurgery, St. Michael's Hospital, 30 Bond Street, Toronto, Ontario, Canada, M5B 1W8. ${ }^{2}$ Labatt Family Centre of Excellence in Brain Injury and Trauma Research, Keenan Research Centre of the Li Ka Shing Knowledge Institute of St. Michael's Hospital, 30 Bond Street, Toronto, Ontario, Canada, M5B 1W8. '3epartment of Surgery, University of Toronto, 30 Bond Street, Toronto, Ontario, Canada, M5B 1W8.

Published: 9 November 2012

\section{References}

1. Ma J, Huang S, Ma L, Liu Y, Li H, You C: Endothelin-receptor antagonists for aneurysmal subarachnoid hemorrhage: an updated meta-analysis of randomized clinical trials. Crit Care 2012, 16:R198.

2. Zimmermann M: Endothelin in cerebral vasospasm. Clinical and experimental results. J Neurosurg Sci 1997, 41:139-151.

3. Roux S, Breu V, Giller T, Neidhart W, Ramuz H, Coassolo P, Clozel JP, Clozel M: Ro 61-1790, a new hydrosoluble endothelin antagonist: general pharmacology and effects on experimental cerebral vasospasm. J Pharmacol Exp Ther 1997, 283:1110-1118.

4. Ikeda S, Awane Y, Kusumoto K, Wakimasu M, Watanabe T, Fujino M: A new endothelin receptor antagonist, TAK-044, shows long-lasting inhibition of both ETA- and ETB-mediated blood pressure responses in rats. J Pharmacol Exp Ther 1994, 270:728-733.

5. Vergouwen MD, Vermeulen M, van GJ, Rinkel GJ, Wijdicks EF, Muizelaar JP, Mendelow AD, Juvela S, Yonas H, terBrugge KG, Macdonald RL, Diringer MN, Broderick JP, Dreier JP, Roos YB: Definition of delayed cerebral ischemia after aneurysmal subarachnoid hemorrhage as an outcome event in clinical trials and observational studies: proposal of a multidisciplinary research 
group. Stroke 2010, 41:2391-2395.

6. Vergouwen MD, Algra A, Rinkel GJ: Endothelin receptor antagonists for aneurysmal subarachnoid hemorrhage: a systematic review and metaanalysis update. Stroke 2012, 43:2671-2676.

7. Moher D, Liberati A, Tetzlaff J, Altman DG: Preferred reporting items for systematic reviews and meta-analyses: the PRISMA statement. BMJ 2009, 339:b2535. doi:10.1186/cc11822

Cite this article as: Macdonald RL: Endothelin antagonists in subarachnoid hemorrhage: what next? Critical Care 2012, 16:171. 